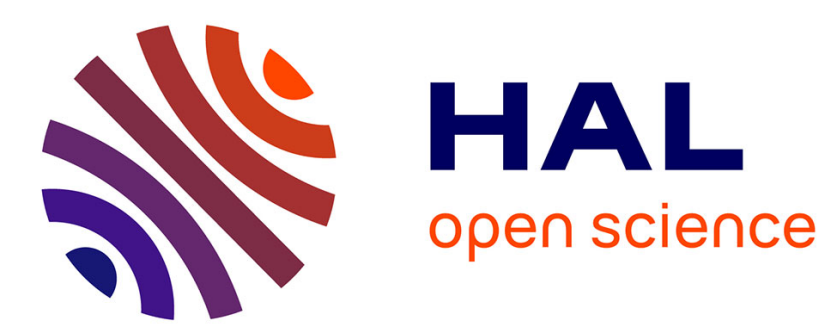

\title{
Geometry-constrained coronary arteries motion estimation from 2D angiograms - Application to injection side recognition
}

\author{
Alice Lebois, Raoul Florent, Vincent Auvray
}

\section{To cite this version:}

Alice Lebois, Raoul Florent, Vincent Auvray. Geometry-constrained coronary arteries motion estimation from 2D angiograms - Application to injection side recognition. 2011 IEEE International Symposium on Biomedical Imaging: From Nano to Macro (ISBI), Mar 2011, Chicago, Illinois, United States. pp.541-544, 10.1109/ISBI.2011.5872464 . hal-00614138

\section{HAL Id: hal-00614138 \\ https://hal.science/hal-00614138}

Submitted on 9 Aug 2011

HAL is a multi-disciplinary open access archive for the deposit and dissemination of scientific research documents, whether they are published or not. The documents may come from teaching and research institutions in France or abroad, or from public or private research centers.
L'archive ouverte pluridisciplinaire HAL, est destinée au dépôt et à la diffusion de documents scientifiques de niveau recherche, publiés ou non, émanant des établissements d'enseignement et de recherche français ou étrangers, des laboratoires publics ou privés. 


\title{
GEOMETRY-CONSTRAINED CORONARY ARTERIES MOTION ESTIMATION FROM 2D ANGIOGRAMS - APPLICATION TO INJECTION SIDE RECOGNITION
}

\author{
Alice Lebois, Raoul Florent and Vincent Auvray
}

\author{
Medisys Research Lab, Philips Healthcare, 33 rue de Verdun, 32156 Suresnes, France
}

\begin{abstract}
This paper deals with the 2D motion estimation of coronary arteries. It exploits the geometry of acquisition to strongly constrain the problem, thereby ensuring smooth and robust motion fields.

The main contribution of this paper is to formally associate the $\mathrm{C}$-arm angulation from which the heart is observed to the expected parametric motion field. Once this learning phase performed, every new sequence is associated to a fixed motion model, known up to a scale and an origin.

The quality of the extracted velocities is visually evaluated over a large representative database. We also present an application of our approach to the motion-based classification of an angiographic exam into right or left artery tree.
\end{abstract}

Index Terms - Coronary arteries, motion estimation.

\section{INTRODUCTION}

Many applications require or benefit from the motion estimation of the coronary arteries. Let us mention the tracking of perfusion basins along the heart cycle [1], or the improvement of reconstruction from a rotational angiography [2]. Depending on the application, it is either desirable to have a strongly regularized (smooth) or a data-driven (accurate) motion field. This topic has been mainly tackled in 3D [3, 4]. However, $3 \mathrm{D}$ data is available in a minority of cases only. This paper presents a robust framework to automatically estimate the motions of the coronary arteries from 2D angiographies.

Our goal is to deliver smooth motion fields (to the possible cost of fine local accuracy), for instance for classification purposes. We are focusing on developing a particularly robust estimation scheme.

It is mandatory for a majority of applications to run without manual interaction. Now, the first step of a motion estimation framework specifically dedicated to the coronary arteries is to extract or enhance the considered objects, yielding vessel maps with many false alarms when no user interaction is allowed. A robust estimation method is needed to extract reliable motion fields out of those noisy maps.

To ensure the required robustness, we propose to constrain the problem by imposing a known parametric model (here, quadratic) to the motion field. Depending on the perspective from which the heart is observed, the 12 parameters defining the motion field are determined and fixed up to a scale and an origin.

Indeed, the 2D observed motions of the coronary arteries is highly correlated to the geometry of acquisition, defined by the two angles of the $\mathrm{C}$-arm. Our main contribution is to formalize this correlation and to exploit it to perform a fast and robust motion estimation of the considered coronary artery tree. We also present an application of our work to the motion-based classification of an angiographic exam into right or left artery.

\section{GEOMETRY-CONSTRAINED PARAMETRIC MOTION ESTIMATION}

The same arteries observed from the same perspective exhibit the same kind of motion. To formalize that assumption, we consider a representative database of 80 angiographies. Fig. 1 show their distribution relative to the two angles $\left(\phi_{1}, \phi_{2}\right)$ defining the acquisition geometry. We observe that they naturally gather in different clusters typical of the recommended reference diagnostic exams.

This section explains how 2D motions representative for each of those clusters can be computed in an offline step (subsection 2.3), and how they can be adapted to a new sequence in an online step (subsection 2.4). The initial vessel map computation is presented in subsection 2.1 .

In this paper, the estimation is performed between the end diastole and the end systole cardiac phases. As a result, in the following, any sequence will be limited to two vessel maps only: $V_{\text {diast }}$ and $V_{\text {syst }}$. This work can of course be applied to any two given instants of the heart cycle.

\subsection{Vessel map computation}

One can hardly rely on the original images $I$ to estimate the motion of the coronary arteries since they are superimposed to the static background [5]. To separate them, we resort to a multiscale ridge filter $R$ [6]. To remove the background linear structures, the minimum value of the previous maps is subtracted from the ridge map:

$$
V(x, y, t)=R(I(x, y, t))-\min _{t^{\prime} \in[0, t-1]} R\left(I\left(x, y, t^{\prime}\right)\right)
$$




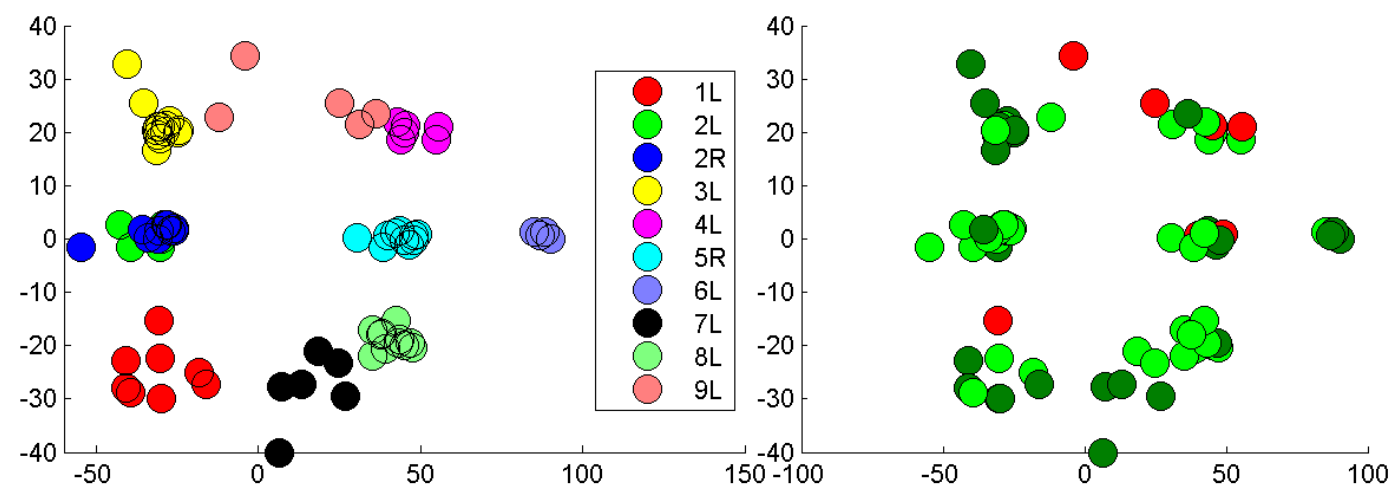

Fig. 1. Database of angiographic sequences as a function of the two C-arm angles $\left(\phi_{1}, \phi_{2}\right)$. Left: gathering into 10 clusters (color-coded), according to the angulation and the observed coronary tree branch (left L or right R). The clusters '2L' and '2R' correspond to right and left coronary exams performed from the same angulations. Right: evaluation of the matching between the estimated velocity fields and the observed motion (light green: excellent; dark green: correct; red: bad).

\subsection{Problem statement}

Our aim being to characterize the visual motion of the coronary arteries, we resort to parametric motion fields since they allow to describe a wide range of motions with a few parameters. We experimentally observed that each cluster $C$ (gathering $N_{C}$ sequences) exhibits a dominant motion that can be described with a quadratic field $\theta_{C}=\left(a_{1}^{C} \ldots a_{12}^{C}\right)$ (12 parameters - Fig.2). We mean that each sequence $S_{i}^{C}$ in the cluster follows the considered motion, up to a scale parameter $\lambda_{i}$ and a translation (defined by the parameter $x_{i}^{0}$ and $y_{i}^{0}$ ). At pixel $(x, y)$, the velocity along $x$ reads:

$$
\begin{aligned}
v_{x}^{S_{i}}(x, y)=\lambda_{i} & \left(a_{1}^{C}+a_{2}^{C}\left(x-x_{i}^{0}\right)+a_{3}^{C}\left(y-y_{i}^{0}\right)\right. \\
& +a_{4}^{C}\left(x-x_{i}^{0}\right)^{2}+a_{5}^{C}\left(y-y_{i}^{0}\right)^{2} \\
& \left.+a_{6}^{C}\left(x-x_{i}^{0}\right)\left(y-y_{i}^{0}\right)\right)
\end{aligned}
$$

The velocity $v_{y}^{S_{i}}$ in $y$ has a similar form.

\subsection{Cluster parameter estimation}

To estimate the parameters $\theta_{C}$ typical of the cluster, we introduce the following energy to be minimized wrt $\theta_{C}$ and the $3 N_{C}$ parameters $\lambda_{i}, x_{i}^{0}$ and $y_{i}^{0}$ :

$$
\sum_{i \in C} \sum_{(x, y) \in S_{i}}\left(V_{\text {syst }}\left(x+v_{x}^{S_{i}}, y+v_{y}^{S_{i}}\right)-V_{\text {diast }}(x, y)\right)^{2}
$$

with $v_{x}^{S_{i}}$ and $v_{y}^{S_{i}}$ following (2). Under the assumption of small motions, the difference of displaced vesselness

$$
V_{\text {syst }}\left(x+v_{x}^{S_{i}}, y+v_{y}^{S_{i}}\right)-V_{\text {diast }}(x, y)
$$

can be linearized as a function of $\delta \theta_{C}$ and $\delta \lambda_{i}$. Solving (3) then boils down to solving a $\left[N_{C}+12 \times N_{C}+12\right]$ linear system. We can therefore jointly estimate the quadratic motion and the $N_{C}$ scales parameter in an efficient multiresolution framework.

It is not acceptable to similarly linearize (4) with respect to $x_{i}^{0}$ and $y_{i}^{0}$, since those parameters often prove to be sizable (possibly $>100$ pixels). We have chosen to perform an exhaustive search to estimate them, at each resolution level.

Finally, the estimation algorithm is the following: at each resolution level

- Update the quadratic $\left(\theta_{C}\right)$ and scale $\left(\lambda_{i}\right)$ parameters by minimizing a linearized version of (3);

- Adjust the translation parameters $x_{i}^{0}$ and $y_{i}^{0}$ by an exhaustive local search.

Since the scales $\left(\lambda_{i}\right)$ and relative shifts $\left(x_{i}^{0}, y_{i}^{0}\right)$ of the sequences in the cluster are estimated in the course of the algorithm, we are able to process a large variety of sequences without imposing constraints to the user (like centering the vasculature, using a given scale...).

\subsection{Sequence parameter estimation}

Once the learning phase performed, every new sequence is associated to a given motion parameter set $\theta_{C}$ based on its angulation. We then simply have to estimate 3 parameters: the scale $\lambda$ and the origin $\left(x_{o}, y_{o}\right)$ of the motion.

We perform a multiresolution estimation as in subsection 2.3, alternatively updating $\lambda$ (analytic expression after linearization) and $\left(x_{o}, y_{o}\right)$ (iterative research).

Depending on the application, the estimated field can be directly used (if a smooth motion is necessary), or can initialize a more accurate motion estimation.

\section{MOTION ESTIMATION RESULTS}

The geometry-constrained motion estimation described in subsection 2.3 has been applied to 80 representative angiographic sequences, split into 10 clusters (left of Fig.1). 
Since the goal is to capture the visual motion, no explicit metric was involved. Instead, we have performed a qualitative assessment. We observed whether the estimated motion matches badly, correctly (the dominant translation as well as the muscle contraction is captured) or excellently (finer motion pattern are also captured).

The overall result is shown on the right of figure 1. Globally, $90 \%$ of the sequences exhibit a correct $(39 \%)$ to excellent match $(51 \%)$ with the visual observation.

Six examples of good matching are shown on Fig.2 a)-f). They present a large variety of anatomies, scale, and vessel visibility. The coronary motions, though different in shape and amplitude, are well captured (including the rotational and shearing components), even though the framework is based on relatively noisy vessel maps.

Motion estimation is incorrect in $10 \%$ of the sequences. In particular, the estimation performs worse in the cluster $5 \mathrm{R}$ (Fig.1). It corresponds to right coronary arteries presenting a complex twisting without clear global motion, which cannot be captured by one single quadratic model (the velocity field is correct on Fig.2 f) (little torsion), but not on Fig.2 g)). We could either introduce a more complex motion model, or consider that it is up to a motion refinement step to capture the observed twisting.

The other poor estimations correspond to isolated sequences in the other clusters. Those sequences present motions that are actually different from the motions observed on the other sequences of the cluster (and indeed they often correspond to angulations at the limits of the considered cluster). More data would be needed to allow for a finer separation of the angulation space in more accurate clusters.

\section{APPLICATION TO INJECTION SIDE RECOGNITION}

The clinician typically observes the right and left coronary arteries successively. Being able to establish which side is being observed has a number of applications. For instance, one could position shutters and wedges according to the ongoing exam, which would reduce the dose.

In our database, 2 of the 10 clusters correspond to right coronary arteries (17/80 sequences): the clusters $2 \mathrm{R}$ and $5 \mathrm{R}$ in Fig.1. If the former can be clearly delineated based on the angulation, the latter corresponds to angulations also used for left coronary exams (cluster 2L).

Since the motions observed in both classes are very different (they correspond to cases a) and b) on Fig.2), we resort to motion to discriminate them. More precisely, we fit both considered quadratic motion models over each sequence using the method presented in 2.4. The class yielding the smallest residual is selected.

This leads to excellent classification performance of $94 \%$ in a leave-one-out evaluation. The one erroneous classification result corresponds to nearly static left coronary. Integrating the expected amplitude of the motion is a perspective to further improve the classification performance.

\section{CONCLUSION AND PERSPECTIVES}

We propose a novel coronary artery estimation method that exploits the strong correlation between the observed motions and the geometry of acquisition. The $\mathrm{C}$-arm angulation space can be split into regions where the expected (quadratic) motion model is known up to a scale and an origin. We report a good matching with the observations, and present a first application of this synthetic motion characterization for classification purposes.

Futhermore, our approach characterizes the observed coronary motion with a small set of parameters. As such, they form excellent features allowing to integrate the global coronary motion in a machine learning framework.

We now need to explore to what extent our approach can improve the robustness and speed of motion estimation methods aiming at a finer accuracy. We intend to measure the accuracy obtained by other methods when the geometric constrained approach reported here is used as initialization.

\section{REFERENCES}

[1] J.Liénard and R.Vaillant, "Quantitative tool for the assessment of myocardial perfusion during X-ray angiographic procedures," in Functional Imaging and Modeling of the Heart FIMH'09, 2009, pp. 124-133.

[2] E.Hansis, D.Schäfer, O.Dössel, and M.Grass, "Projection-based motion compensation for gated coronary artery reconstruction from rotational X-ray angiograms," Physics in Medicine and Biology, vol. 53, no. 14, pp. 3807-3820, June 2008.

[3] C.Blondel, G.Malandrain, R.Vaillant, and N.Ayache, "Reconstruction of coronary arteries from a single rotational X-ray projection sequence," IEEE Transaction on Medical Imaging, vol. 25, pp. 653-663, 2006.

[4] D.Schäfer, J.Borgert, V.Rasche, and M.Grass, "Motioncompensated and gated cone beam filtered backprojection for 3-d rotational X-ray angiography," IEEE Trans. on Medical Imaging, vol. 25, pp. 898-906, 2006.

[5] V.Auvray, P.Bouthemy, and J.Liénard, "Joint motion estimation and layer segmentation in transparent image sequences - application to noise reduction in X-ray image sequences," EURASIP Journal on Advances in Signal Processing, 2009.

[6] A.F. Frangi, W.J. Niessen, K.L. Vincken, and M.A. Viergever, "Multiscale vessel enhancement filtering," in LNCS(1496), MICCAI’98, 1998, pp. 130-138. 

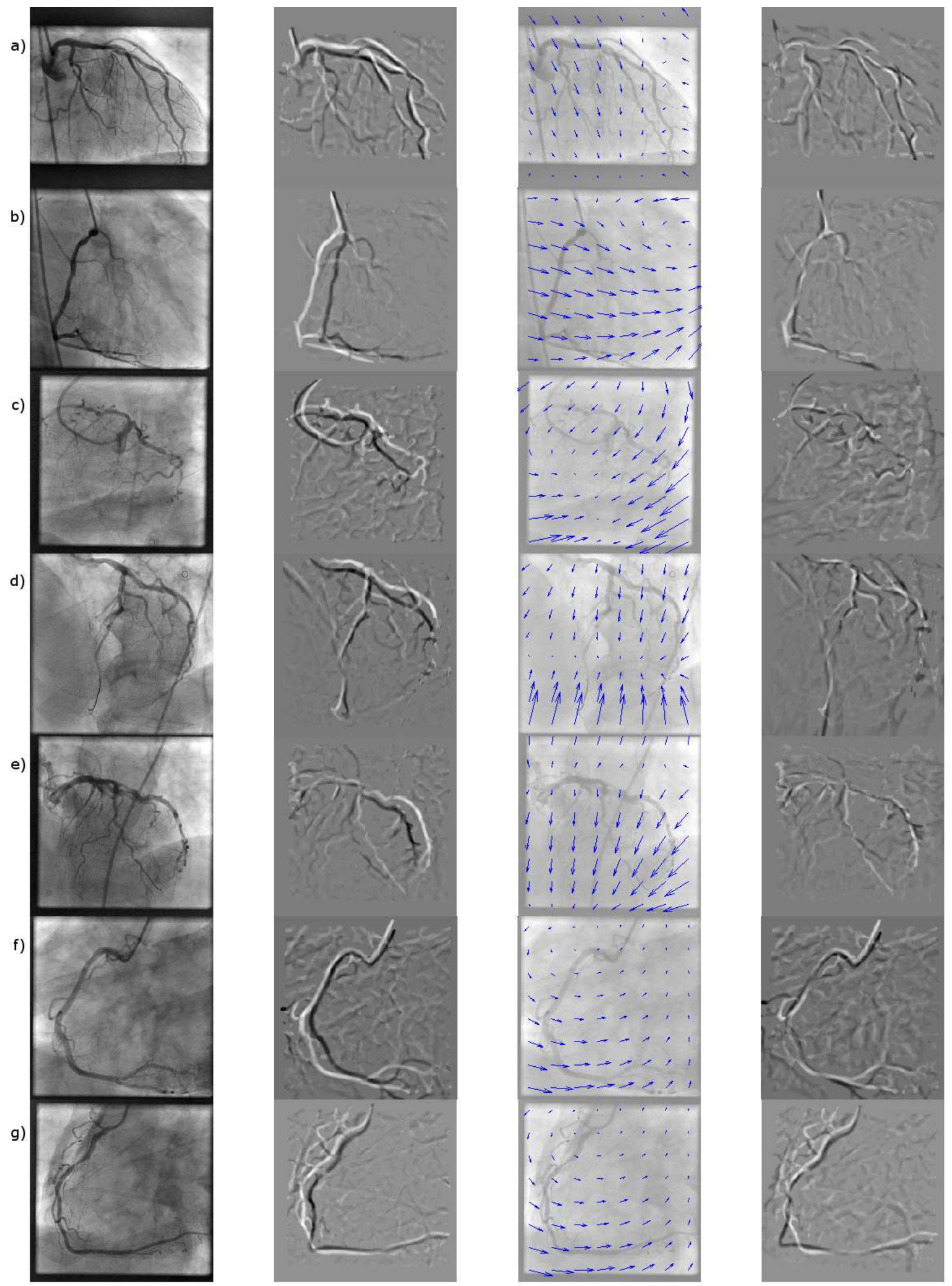

Fig. 2. Different sequences together with the estimated generic field. From left to right: coronary artery at diastole; noncompensated vesselmap difference (illustrating the shape and the amplitude of the motion), estimated motion field (with the proper center and scale), motion-compensated vesselmap difference (illustrating the quality of the motion estimation). Clusters a) $2 \mathrm{~L} \mathrm{~b}) 2 \mathrm{R}$ c) $4 \mathrm{~L} \mathrm{~d}$ ) $8 \mathrm{~L} \mathrm{e)} 8 \mathrm{~L} \mathrm{f}$ ) $5 \mathrm{R} \mathrm{g}$ ) $5 \mathrm{R}$ (see clusters on Fig.1). 